\title{
An Improvement of Magnetic Flux Linkage in Electrical Generator using the novel Permanent Magnet Arrangement
}

\begin{abstract}
W. Sriwannarat ${ }^{a}$, P. Khunkittit ${ }^{a}$, A. Janon ${ }^{b}$ And A. Siritaratiwat ${ }^{a, *}$
${ }^{a}$ Department of Electrical Engineering, Faculty of Engineering, Khon Kaen University, Khon Kaen 40002, Thailand ${ }^{b}$ Department of Mechanical Engineering, Faculty of Engineering, Khon Kaen University, Khon Kaen 40002, Thailand

The partitioned stator permanent magnet generator (PS-PMG) has been extensively used as electrical generator due mainly to their high flux linkage, high electromotive force EMF) and high efficiency, especially the PM arrangement of the PS-PMG is an important factor to improve the performance of the generator. Therefore, we propose the novel permanent magnet PM) arrangement of the PS-PMG by using the dual rotor with coaxial core structure in order to improve the flux linkage and the EMF of PS-PMG. The PM position of stators of the conventional PS-PMG was rearranged, then the electrical characteristic of the proposed structure was characterized through the simulations based on finite element method. It was found that the flux linkage produced by the proposed structure is $37.06 \%$ higher than that of the conventional PS-PMG existed in the literatures because the area for induced flux linkage is significantly increased. This flux linkage enhancement further causes an increment of EMF about 34.95\% compared to that of the conventional PS-PMG under the same condition. Especially, the EMF produced by the proposed structure is classified in the high-value range regarding the PM generator. Then, the PS-PMG using novel permanent magnet arrangement could be another remarkable choice for the electrical generator application
\end{abstract}

DOI: 10.12693/APhysPolA.133.642

PACS/topics: 03.50.De, 07.55.Db, 75.50.Ww, 83.60.Np, 84.30.Ng, 85.70.Ay

\section{Introduction}

The permanent magnet $(\mathrm{PM})$ machines based on NdFe-B have been extensively used as the electrical generator since it could produce high flux linkage and high electromotive force (EMF). Also, these PM based generators are classified in high efficiency electrical generators due mainly to their no field excitation and no copper loss characteristics [1]. The stator-PM machines are categorized into three types which are the flux-reversal PM machine, the switched-flux PM machine and the doublysalient PM machine $[2,3]$. Recently, several studies show that the back electromotive force (EMF) and the torque density of the stator-PM machines can be significantly improved by using the partitioned stator (PS) technique, which is appropriate to be used as the electrical generator application [4-6].

In addition, the PM arrangement of the partitioned stator PM generator (PS-PMG) has been an important factor that significantly affects the flux linkage and EMF of this generator, which indicates as the major indicator regarding the machine performance $[7,8]$. Normally, the magnetic flux distribution of conventional PS-PMG is circulated only through the armature winding located at the outer stator, however the flux linkage can be increasingly improved by a modification of PM arrangement in the generator structure.

Then, the aim of this work is to propose a novel permanent magnet arrangement of the partitioned stator

\footnotetext{
*corresponding author; e-mail: apirat@kku.ac.th
}

PM generator (PS-PMG) by using the dual rotor with coaxial core structure in order achieve higher flux linkage and EMF. The simulations were based on Finite Element Method.

\section{Topology selection}

As shown in Fig 1(a), the simulation model of $12 / 10$ (stator/rotor) conventional PS-PMG is adopted from J.T. Shi et al. [5]. It comprises the outer armature winding stator and inner PM stator with separated by the rotor. Then, we propose the novel permanent magnet arrangement by installing the additional inner armature winding stator and inner rotor to the structure while using the common axial core, the proposed structure is defined as the partitioned stator permanent magnet generator with dual rotor coaxial core (PS-PMG-DR), as shown in Fig 1(b). The proposed PS-PMG-DR consists of outer-, inner-stator with concentrated armature winding and middle-stator with PM mounted on the surface. The outer- and inner-rotor are sandwiched between three stators. The PM stator is based on Nd-Fe-B material where the number of PM is equal to the number of stator poles. The parameters of PS-PMG and PS-PMG-DR structures are detailed in Table I.

\section{Results and discussion}

\subsection{Magnetic flux distribution analysis}

The magnetic flux distribution of the proposed PSPMG-DR was firstly analyzed, and then it was compared to that of the conventional PS-PMG. As shown 


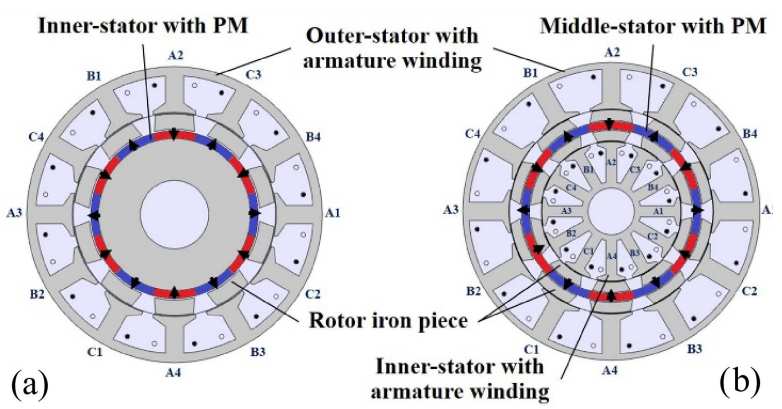

Fig. 1. A 3-phase cross section $12 \mathrm{~S} / 10 \mathrm{R}$ of a) PS-PMG and $\mathrm{b}$ ) proposed PS-PMG-DR.

TABLE I

The parameters of PS-PMG and PS-PMG-DR.

\begin{tabular}{|c|c|c|}
\hline Parameter & PS-PMG & PS-PMG-DR \\
\hline Outer stator (OS) pole number & \multicolumn{2}{|c|}{12} \\
\hline Inner stator (IS) pole number & \multicolumn{2}{|r|}{12} \\
\hline Rotor pole number & \multicolumn{2}{|r|}{10} \\
\hline Number of PM & \multicolumn{2}{|r|}{12} \\
\hline PM inner pole arc $\left[{ }^{\circ}\right]$ & \multicolumn{2}{|r|}{30} \\
\hline PM thickness [mm] & \multicolumn{2}{|r|}{3.5} \\
\hline Magnetic remanence $[\mathrm{T}]$ & \multicolumn{2}{|c|}{1.20} \\
\hline Number of phases & \multicolumn{2}{|c|}{3} \\
\hline Number of turn & \multicolumn{2}{|r|}{1} \\
\hline Rate speed [rpm] & \multicolumn{2}{|r|}{400} \\
\hline Air gap length [mm] & \multicolumn{2}{|r|}{0.5} \\
\hline OS outer radius [mm] & \multicolumn{2}{|c|}{66.5} \\
\hline IS inner radius [mm] & 15.37 & 10.4 \\
\hline OS inner radius $[\mathrm{mm}]$ & 45.89 & 20.7 \\
\hline IS outer radius [mm] & 37.62 & 20.7 \\
\hline OS tooth body pole arc $\left[{ }^{\circ}\right]$ & \multicolumn{2}{|c|}{10.2} \\
\hline OS tooth tip pole $\operatorname{arc}\left[{ }^{\circ}\right]$ & \multicolumn{2}{|c|}{6.9} \\
\hline OS tooth tip thickness open [mm] & 1.48 & 1 \\
\hline OS tooth tip thickness body [mm] & 4.44 & 2 \\
\hline Rotor outer pole $\operatorname{arc}\left[{ }^{\circ}\right]$ & \multicolumn{2}{|r|}{22} \\
\hline Rotor inner pole arc $\left[^{\circ}\right]$ & \multicolumn{2}{|c|}{27.2} \\
\hline Rotor radial thickness [mm] & 6.8 & 4.6 \\
\hline OS yoke thickness [mm] & 4.14 & 2.8 \\
\hline IS tooth body pole arc $\left[{ }^{\circ}\right]$ & - & 10.2 \\
\hline IS tooth tip pole $\operatorname{arc}\left[{ }^{\circ}\right]$ & - & 6.9 \\
\hline IS tooth tip thickness open [mm] & - & 1 \\
\hline IS tooth tip thickness body [mm] & - & 2 \\
\hline OS yoke thickness [mm] & - & 2.8 \\
\hline
\end{tabular}

in Fig 2, a comparison of open-circuit magnetic flux distribution between the PS-PMG and PS-PMG-DR is indicated in case of the same size of generator. It was observed that the initial configuration of magnetic flux distribution of PS-PMG and proposed PS-PMG-DR is circulated through three poles at upper and lower armature winding of outer-stator. Especially, the magnetic flux of PS-PMG-DR can be also circulated through an additional armature winding of the inner-stator, which indicates as an increase of the area for induced flux linkage. As a result, the magnetic flux distribution of the proposed PS-PMG-DR could further improve the flux linkage magnitude of the conventional PS-PMG.

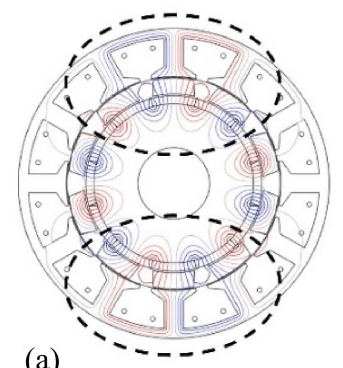

(a)

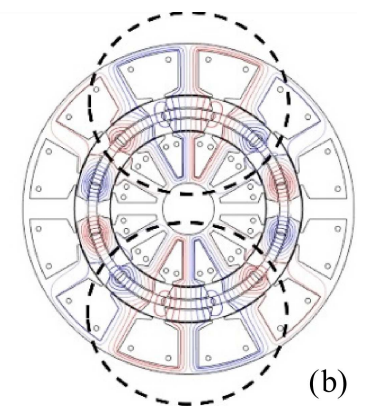

(b)
Fig. 2. Open circuit magnetic flux distributions of the a) PS-PMG and b) proposed PS-PMG-DR.

\subsection{Influence of PM pole arc and thickness analysis}

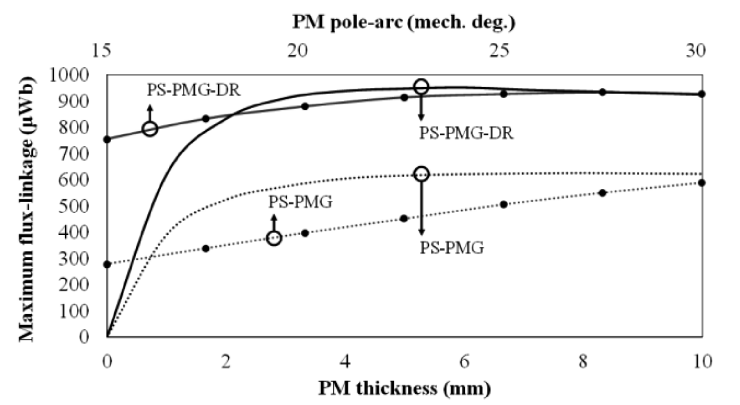

Fig. 3. A variation of the maximum flux linkage in the PS-PMG and PS-PMG-DS with various: PM pole arcs at the PM thickness of $3.5 \mathrm{~mm}$ and PM thickness at PM pole arc $30^{\circ}$.

The maximum flux linkage of PS-PMG and PS-PMGDS with the PM pole-arc of 15-30 degrees at a constant PM thickness of $3.5 \mathrm{~mm}$ is shown in Fig 3. It was found that the maximum flux linkage of both structures is increased at higher PM pole arc degrees. The maximum flux linkage of PS-PMG-DR tends to be stable when the $\mathrm{PM}$ pole arc degree of $30^{\circ}$ is reached since the width of the tip of outer- and inner-stator are appropriate for PM pole arc. Then, the effects of PM thickness on the maximum flux linkage will be further investigated at the pole arc of 30 degree.

As shown in Fig. 3, the maximum flux linkage of PSPMG and PS-PMG-DR at various PM thicknesses of 0 $10 \mathrm{~mm}$ with a step size of $1 \mathrm{~mm}$ is examined at a constant PM thickness of 30 degree. It was found that the maximum flux linkage of both structures is significantly increased at the PM thickness of $04 \mathrm{~mm}$ since the additional PM thickness directly enhances the magnetic flux distribution. After that, it was gradually stable due to the magnetic flux saturation at the PM thickness of 56 
mm. Then,the flux linkage was slightly decreased at the PM thickness of 7-10 mm because of the flux leakage. Therefore, it is apparently seen that the PS-PMG-DR could produce higher flux linkage than the conventional PS-PMG at all PM pole arcs and PM thicknesses because the area for induced flux linkage is increased by the additional inner stator and inner rotor.

\subsection{Phase flux linkage and EMF analysis}

In addition, the phase flux linkage of the PS-PMG-DR was characterized and compared to that of the conventional PS-PMG As shown in Fig. 4, a comparison between the phase flux linkage of the PS-PMG and PSPMG-DR is illustrated. The symmetrical property was observed in the flux linkage waveform obtained from both generators. The maximum flux linkages of 588.47 and 935.02 $\mu \mathrm{Wb}$ were found for PS-PMG and PS-PMG-DR, respectively. It was found that the flux linkage produced by the PS-PMG-DR is higher than that of the PS-PMG at all rotor positions. This is because the proposed PSPMG-DR has an additional inner rotor and armature winding inserted to the inner stator, which typically increases the area for flux linkage induction yielding an enhancement of the flux linkage.

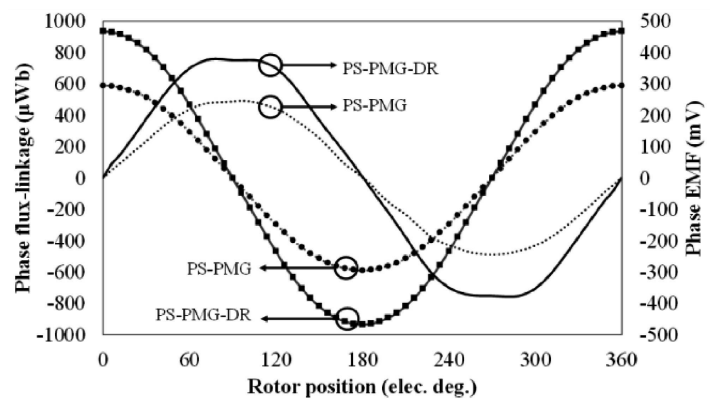

Fig. 4. The open circuit phase flux linkage and phase EMF of PS-PMG and PS-PMG-DR.

The phase EMF of the PS-PMG and PS-PMG-DR was in addition examined, as shown in Fig. 4. It was found that the phase EMF of PS-PMG-DR is higher than that of the PS-PMG at all rotor positions, which is consistent with the characteristic of the flux linkage. The highest EMF of $229.22 \mathrm{mV}$ was found for the conventional PSPMG, where that of $365.69 \mathrm{mV}$ was found for PS-PMGDR.

From the results, it is noticed that the proposed PSPMG-DR can increases the magnetic flux linkage and EMF of the generator due to a modification permanent magnet arrangement which significantly expands the induced magnetic flux area. Especially, the EMF found in this proposed PS-PMG-DR is classified in the high value range compared to the other types of PM generators existed in the literature. An enhancement of the flux linkage and EMF found in this proposed PS-PMGDR could also improve the electromagnetic performance of electrical generator. Hence, the PS-PMG-DR is indicated as another special choice for electrical generator in the future.

\section{Conclusions}

The novel permanent magnet arrangement of PS-PMG was proposed by using the dual rotor with coaxial core structure. The flux linkage and the EMF of the novel structure were characterized and compared to that of the conventional PS-PMG. It was verified that the magnetic flux induction of PS-PMG-DR was significantly improved from the conventional PS-PMG due to the adjusted permanent magnet arrangement. The phase flux linkage and EMF of PS-PMG-DR were improved from the conventional PS-PMG by $37.06 \%$ and $34.95 \%$, respectively, which typically improves the electrical performance of the generator. Therefore, the PS-PMG-DR becomes another remarkable choice for the electrical generator application.

\section{Acknowledgments}

This work was financially supported by the center for Alternative Energy Research and development with the Applied Mathematics and Optimization Research Unit. Author would also like to thank the Department of Mechanical Engineering, Khon Kaen University for COMSOL software facility.

\section{References}

[1] M. Kazmierczak, P. Pawlik, J.J. Wysłocki, I. Wnuk, P. Gebara, K. Pawlik, A. Przybył, Acta Phys. Pol. A 131, 789 (2017)

[2] C. Ming, H. Wei, Z. Jianzhong, Z. Wenxiang, IEEE Trans. Ind. Electr. 58, 11, 50875101 (2011).

[3] A. Jadallah, D. Mahmood, Z. Er, Z. Abdulqaedr, Przybył, Acta Phys. Pol. A 130, 434 (2016).

[4] Z.Q. Zhu, Z.Z Wu, D.J. Evans, W.Q. Chu, IEEE Trans Magn. 51, 19 (2015).

[5] Z.Z Wu, Z.Q. Zhu, J.T. Shi, IEEE Trans. Magn. 51, 8105212 (2015).

[6] D.J. Evans, Z.Q. Zhu, IEEE Trans. Magn. 51, 114 (2015).

[7] Z.Q. Zhu, H. Hua, D. Wu, J.T. Shi, Z.Z. Wu, IEEE Trans. Ind. Appl. 52, 1, 199208 (2016).

[8] J.T. Shi, A.M. Wang, Z.Q. Zhu, IEEE Trans. Magn. 53, $112(2017)$. 